\title{
Complicações dos métodos de revascularização cardíaca em pacientes que sofreram infarto agudo do miocárdio
}

\author{
Complications of cardiac revascularization methods in patients who suffered acute \\ myocardial infarction
}

Complicaciones de los métodos de revascularización cardíaca en pacientes que sufrieron infarto agudo de miocardio

Tom Ravelly Mesquita Costa ${ }^{1 *}$, Paulo César Monteiro Florêncio', Ana Luíza de Castro Carvalho², Bianca Araujo Ruiz ${ }^{3}$, Cícero Santos Souza ${ }^{4}$, Karolinne Kassia Silva Barbosa ${ }^{5}$, Marília Guedes Farias Barbosa $^{6}$, Priscylla Frazão Rodrigues ${ }^{1}$, Ingrid Cristina Rêgo Barros ${ }^{1}$, Marcos Aurélio Lima Barros ${ }^{1}$.

\section{RESUMO}

Objetivo: Identificar as complicações apresentadas por pacientes Pós-Infarto Agudo do Miocárdio (IAM), submetidos a diferentes métodos de revascularização cardíaca como opção terapêutica. Métodos: Trata-se de uma revisão sistemática por meio dos indexadores nas bases de dados eletrônicos Biblioteca Virtual em Saúde (BVS), Medical Literature Analysis and Retrieval System Online (MEDLINE) e Web of Science. Além disso, foi realizado o cálculo do coeficiente de kappa (k) e a escala de Jadad. Resultados: Durante a pesquisa 16 artigos foram selecionados. Observou-se que os principais métodos utilizados foram a Intervenção Coronária Percutânea (IPC) e a Cirurgia de Revascularização Miocárdica (CRVM). No que se refere às complicações verificadas, constatou-se que o IAM foi a complicação mais prevalente, seguido do Acidente Vascular Cerebral (AVC), eventos hemorrágicos, morte pós-operatória e a morte cardíaca. Considerações Finais: Por fim, identificou-se as complicações apresentadas por pacientes Pós-Infarto Agudo do Miocárdio (IAM), submetidos a diferentes métodos de revascularização, se apresentando como fibrilação atrial, morte súbita, disritmias ventriculares, insuficiência cardíaca, processos infecciosos e infarto do miocárdio, além do Acidente Vascular Cerebral (AVC) e eventos hemorrágicos seguidos por morte pós-operatória. Portanto, é imprescindível novas técnicas, alcançando um tratamento adequado.

Palavras-chave: Complicações, Infarto do miocárdio, Revascularização miocárdica, Ensaios clínicos.

\section{ABSTRACT}

Objective: To identify the complications presented by patients after acute myocardial infarction (AMI), submitted to different methods of cardiac revascularization as a therapeutic option. Methods: This is a systematic review using indexers in the electronic databases Virtual Health Library (VHL), Medical Literature Analysis and Retrieval System Online (MEDLINE) and Web of Science. In addition, the kappa coefficient (k) and the Jadad scale were calculated. Results: During the research, 16 articles were selected. It was observed that the main methods used were Percutaneous Coronary Intervention (IPC) and Myocardial Revascularization Surgery (CABG). Regarding the complications observed, it was found that AMI was the most prevalent complication, followed by stroke, hemorrhagic events, postoperative death and cardiac death. Final Considerations: Finally, we identified the complications presented by Post-Acute Myocardial Infarction (AMI) patients, submitted to different revascularization methods, presenting as atrial fibrillation, sudden death, ventricular dysrhythmias, heart failure, infectious processes and infarction myocardial infarction, in addition to stroke and hemorrhagic events followed by postoperative death. Therefore, new techniques are essential, achieving adequate treatment.

Keywords: Complications, Myocardial Infarction, Myocardial revascularization, Clinical trials.

\footnotetext{
1 Universidade Federal do Delta do Parnaíba (UFDPar), Parnaíba - PI. *E-mail:tomravelly@gmail.com

2 Universidade Federal de Juiz de Fora (UFJF), Juiz de Fora - MG.

${ }^{3}$ Centro Universitário Nilton Lins (UNL), Manaus - AM.

${ }^{4}$ Universidade Estadual do Sudoeste da Bahia (UESB), Jequié - BA.

${ }^{5}$ Instituto de Educação Superior do Vale do Parnaíba (IESVAP), Parnaíba - PI.

${ }^{6}$ Centro Universitário Maurício de Nassau (UNINASSAU), Recife - PE.
} 


\section{RESUMEN}

Objetivo: Identificar las complicaciones que presentan los pacientes tras un infarto agudo de miocardio (IAM), sometidos a diferentes métodos de revascularización cardíaca como opción terapéutica. Métodos: Se trata de una revisión sistemática utilizando indexadores en las bases de datos electrónicas Virtual Health Library (BVS), Medical Literature Analysis and Retrieval System Online (MEDLINE) y Web of Science. Además, se calculó el coeficiente kappa (k) y la escala de Jadad. Resultados: Durante la investigación se seleccionaron 16 artículos. Se observó que los principales métodos utilizados fueron la Intervención Coronaria Percutánea (IPC) y la Cirugía de Revascularización Miocárdica (CABG). En cuanto a las complicaciones observadas, se encontró que el IAM fue la complicación más prevalente, seguida del ictus, eventos hemorrágicos, muerte postoperatoria y muerte cardíaca. Consideraciones finales: Finalmente, identificamos las complicaciones que presentan los pacientes postinfarto agudo de miocardio (IAM) sometidos a diferentes métodos de revascularización, presentándose como fibrilación auricular, muerte súbita, arritmias ventriculares, insuficiencia cardíaca, procesos infecciosos e infarto. infarto de miocardio, además de accidente cerebrovascular y eventos hemorrágicos seguidos de muerte postoperatoria. Por tanto, las nuevas técnicas son fundamentales, consiguiendo un tratamiento adecuado.

Palabras clave: Complicaciones, Infarto de miocardio, Revascularización miocárdica, Ensayos clínicos.

\section{INTRODUÇÃO}

As Doenças Cardiovasculares (DCV) são as principais causas de mortes no mundo e responsáveis também por provocarem danos irreversíveis, como limitações e dependências, as quais influenciam diretamente na qualidade de vida dos pacientes (NASCIMENTO BR, et al., 2018). Dentre os inúmeros fatores de risco associados, têm-se os modificáveis e os não-modificáveis.

Os fatores de riscos modificáveis incluem tabagismo, obesidade, hiperlipidemia, uso de contraceptivos orais, etilismo, hiperglicemia, sedentarismo e má alimentação, já os não modificáveis envolvem a história familiar de doenças pregressas, idade, sexo e raça (MAGALHÃES FJ, et al., 2014).

A Doença Arterial Coronariana (DAC), comum entre as enfermidades cardiovasculares, é o resultado da obstrução das artérias coronárias. A patologia consiste em uma pequena lesão, capaz de acumular ao longo do tempo quantidades consideráveis de lipídios, plaquetas e, consequentemente, auxilia na formação de uma fina camada de fibrose que, posteriormente, torna-se uma placa de ateroma (DE CARVALHO ACA, et al., 2010).

Uma das opções de tratamento para DAC é por meio da Intervenção Coronariana Percutânea (ICP), que consiste na remodelação da placa aterosclerótica de maneira que a lesão obstrutiva se transforme em lesão não obstrutiva estável (DI CASTRO FAM, et al., 2016). É o método mais comumente empregado para revascularização coronariana, superando a cirurgia e aliviando a angina, com a melhora da qualidade de vida e a redução de mortalidade nos casos agudos (PIEGAS LS, 2015; HADDAD N, 2011).

A eventual ruptura da placa, leva o desenvolvimento de um trombo, manifestando-se clinicamente de inúmeras formas, a depender de sua extensão, podendo apresentar complicações, como uma angina instável ou casos mais graves, como um Infarto Agudo do Miocárdio (IAM), assim, torna-se uma das mais importantes e graves doenças cardiovasculares (SIERVULI MTF, et al., 2014).

O IAM é definido como a morte de um segmento do músculo cardíaco decorrente de um desequilíbrio entre oferta e demanda de oxigênio no miocárdio, seu tratamento deve ser efetuado logo no início dos sintomas, por meio de uma assistência pré-hospitalar que tem como principal objetivo a redução do tempo entre o início do evento isquêmico até o tratamento efetivo, restaurando a perfusão miocárdica e reduzindo o mecanismo mais frequente de morte, que é a Fibrilação Ventricular (FV) (ANDRADE JP, et al., 2009).

Após o atendimento pré-hospitalar, inicia-se o processo de diagnóstico e estratificação de risco, logo procedendo a intervenção, mediante o uso de medidas medicamentosas e/ou terapias de reperfusão. Vale ressaltar que, entre as terapias de reperfusão, tem-se a Cirurgia de Revascularização Cardíaca, a qual pode ser utilizada como terapia de urgência e de forma eletiva ((DE SOUZA AR, et al., 2018; PIEGAS LS, et al., 2015). 
Ademais, Cirurgia de Revascularização Miocárdica (CRM), como terapia de urgência, é indicada nos seguintes episódios: revascularização primária na vigência de episódio de infarto; revascularização após ICP sem sucesso; revascularização por angina recorrente; e revascularização associada à correção das complicações mecânicas do infarto. Destaca-se ainda o seu uso como terapia eletiva, sendo indicada em casos em pacientes que apresentaram IAM, geralmente, considerada em um grupo restrito de pacientes com anatomia de artérias coronárias não favoráveis a tratamento com ICP, como também na presença de isquemia recorrente e no comprometimento importante da função ventricular (PIEGAS LS, et al., 2015).

Em vista do atual cenário, com a inversão da pirâmide etária, ocorre um aumento do grupo populacional com faixa etária acima dos 65 anos de idade (MIRANDA GMD, et al., 2016). O avanço significativo associado à exposição aos fatores de risco para o desenvolvimento de doenças do sistema cardiovascular, exige das ciências médicas avanços tecnológicos, assim como das técnicas cirúrgicas. Entretanto, vale lembrar que, mesmo com a presença de avanços em técnicas cirúrgicas, ainda existem fatores de riscos intrínsecos aos próprios pacientes, como é o caso da faixa etária avançada, devendo-se realizar uma avaliação criteriosa nesse grupo, antes de qualquer indicação de cirurgia de revascularização do miocárdio, visando proporcionar uma recuperação mais eficaz e segura, desse modo, interferindo minimamente nos aspectos relacionados à qualidade de vida (DE SOUZA AR, et al., 2018).

Dentre as técnicas cirúrgicas, o método CRM destaca-se por ser eficiente no alívio sintomático e no aumento da expectativa de vida, com a melhora a revascularização do músculo cardíaco, mediante a inserção de uma artéria ou veia saudável do corpo do próprio paciente, sendo introduzida na artéria coronária obstruída (BRICK AV, et al., 2004).

Ocorre que, segundo as Diretrizes da Cirurgia de Revascularização Miocárdica, a artéria ou veia enxertada ignora o bloqueio da artéria coronária, e permite a efetiva oxigenação, ocorrendo o alívio nos sintomas anginosos, com a consequente estabilização do paciente e a redução dos agravos. Em casos de vítimas de IAM, a terapêutica é realizada de acordo com a extensão dos danos no músculo cardíaco, como também pela avaliação de risco cardiovascular. Entretanto, para a tomada de decisão e correta intervenção é preciso, primeiramente, avaliar o paciente quanto aos possíveis riscos e benefícios, em razão de tratar-se de um procedimento cirúrgico, podendo apresentar complicações futuras, intimamente ligadas as condições, como: idade, afecções pulmonares, função cardíaca, doenças metabólicas e insuficiência renal crônica (IRC) (FEITOSA ACR, et al., 2011).

Além disso, como forma de padronizar o tratamento dessas complicações, a Sociedade Brasileira de Cardiologia (SBC) disponibiliza diretrizes sobre os métodos terapêuticos, visando, desta forma, reduzir a necrose do músculo cardíaco (AVEZUM Á, et al., 2004)

A CRM foi incorporada nos procedimentos cirúrgicos do Brasil no início da década de 70 e atualmente é considerada uma das cirurgias mais realizadas no Sistema Único de Saúde (SUS). Ademais, o seu uso no tratamento da DAC, em sua maioria, pode ser realizado em mais de uma artéria coronária. Entre os pacientes que sofreram IAM, cerca de $60 \%$ apresentam estenose de uma ou mais artérias coronárias, além da artéria comprometida (MEHTA SR et al., 2019). Tal fato propõe que a realização da cirurgia de revascularização completa, ou seja, de todas as artérias estenosadas, é capaz de melhorar a angina, expondo o paciente a um único tipo de procedimento, assim, evitando complicações nas demais artérias estenosadas e um outro IAM (HINOHARA TT, et al., 2018).

As enfermidades cardiovasculares, estão entre os principais problemas de saúde pública do Brasil, logo que, no intervalo de idade de 30 a 69 anos, as DCV foram responsáveis por $65 \%$ do total de óbitos no país (GODOY MF, et al., 2007). Estima-se que a taxa de mortalidade secundária a problemas cardiovasculares no Brasil é cerca de (183,3/100.000) (SANTOS J, et al., 2018). Estudos evidenciam um aumento progressivo da mortalidade causada por IAM, com avançar da idade, em ambos os sexos com maiores taxas de mortalidade em homens e em todas as regiões do Brasil (SANTOS J, et al., 2018). Desta forma, estima-se que, a partir do ano de 2020, com o aumento significativo contínuo, tal doença será o principal responsável por causar mortes no país (MIRANDA GMD, et al., 2016). 
A grande parte dos pacientes que sofreram infarto, segundo a literatura, apresentavam, previamente, doenças crônicas, estando as principais: a Hipertensão Arterial Sistêmica (HAS) e o Diabetes Mellitus (DM), porém, dentre todos os aspectos, a idade é o mais prevalente fator de risco para a ocorrência de óbito e contratempos durante a CRM (NASCIMENTO BR, et al., 2018). Desta maneira, é imprescindível compreender as complicações secundárias a esses tipos de procedimentos para que, assim, ocorra de maneira correta a abordagem, e, consequentemente, a diminuição das taxas de complicações.

Frente ao exposto, e partindo da temática do estudo, objetivou-se identificar, mediante a revisão sistemática deste estudo, as complicações apresentadas por pacientes Pós-IAM, submetidos a diferentes métodos de revascularização cardíaca, como opção terapêutica. Decerto, a realização da pesquisa torna-se de cunho importante para o entendimento dos métodos de intervenções cirúrgicas cardíacas, além da possibilidade do aumento de conhecimento dos possíveis contratempos decorridos dos procedimentos.

\section{MÉTODOS}

Trata-se de um estudo do tipo revisão sistemática, que busca identificar as complicações apresentadas por pacientes pós-infarto agudo do miocárdio (IAM), submetidos a diferentes métodos de revascularização cardíaca. Para a produção desta revisão, foi realizado a seleção de artigos hospedados em três bases de dados bibliográficas, BVS, MEDLINE e Web of Science, no intuito de analisar as complicações das cirurgias de revascularização cardíaca em pacientes que sofrem infarto agudo do miocárdio. A coleta de dados e o levantamento bibliográfico foram realizados no mês de junho de 2020.

Para tal levantamento, os Descritores em Ciências da Saúde (DeCS) utilizados foram: Complicações, Infarto do Miocárdio, Revascularização Miocárdica e Ensaios clínicos, e para a busca em bases de dados internacionais utilizou-se como descritores: Myocardial Revascularization, Myocardial Infarction e Clinical trials, baseados no Mesh (Medical Subject Headings) acrescidos do termo Complications. Para o aprimoramento da busca foi utilizado o operador lógico booleano "AND", para relacionar termos, e selecionados artigos de relevância ao tema.

Os dados foram analisados por meio de tabelas e discutidos com a literatura atual, pertinente ao tema. Os artigos foram selecionados de acordo com os seguintes critérios de inclusão: artigos publicados em periódicos nacionais e internacionais, disponibilizados em inglês, espanhol ou português, publicados entre 01 de janeiro de 2010 a 31 de dezembro de 2019, do tipo ensaio clínico randomizado duplo cego, com a finalidade de garantir um bom nível de evidências, as quais versavam sobre a temática complicações das cirurgias de revascularização cardíaca em pacientes que sofrem infarto agudo do miocárdio.

Como critérios de exclusão, foram estabelecidos: estudos com títulos não relacionados diretamente ao tema da pesquisa; estudos com resumos portando dados irrelevantes à pesquisa, como também inapropriado ao tema proposto ou pesquisas discordantes de ensaios clínicos. A seleção dos estudos foi realizada de forma independente por dois pesquisadores com experiência em revisões, a partir da análise dos títulos, resumos e textos completos das publicações. As divergências foram resolvidas por consenso e, na sua impossibilidade, por um terceiro pesquisador com experiência no tema. O cálculo do coeficiente de kappa $(k)$ foi utilizado para demonstrar a concordância ajustada entre os examinadores, considerando-se um valor acima de 0,80 como uma concordância praticamente perfeita (CANTOR AB, 1996).

Para avaliar a qualidade dos estudos selecionados, a escala de Jadad foi utilizada, considerando quatro e cinco escores de boa qualidade, dois e três escores de média qualidade, um e zero escores de baixa qualidade. Os artigos selecionados deveriam ter pontuação igual ou superior a 4. Esta pontuação leva em consideração: um ponto para estudo randomizado, um ponto se a randomização foi feita adequadamente, um ponto se o estudo é duplo-cego, um ponto se o método duplo-cego foi descrito e é apropriado e um ponto se são descritas as perdas, como também exclusões (JADAD AR, et al., 1996).

Dos estudos incluídos, foram coletados e registrados, em um banco de dados para esta pesquisa, as informações sobre: objetivo, população, métodos cirúrgicos utilizados, complicações intraoperatórias, perioperatórias e o desfecho clínico dos pacientes. Os resultados foram sumarizados e apresentados no formato de tabelas. Ademais, a seleção dos artigos prosseguiu de acordo com os critérios propostos pela metodologia do fluxograma desenvolvido pelo PRISMA (Preferred Reporting Items for Systematic Reviews and Meta-Analyses) (Figura 1). 
Figura 1 - Organograma de Busca e seleção dos artigos de acordo com o PRISMA. Brasil, 2020.

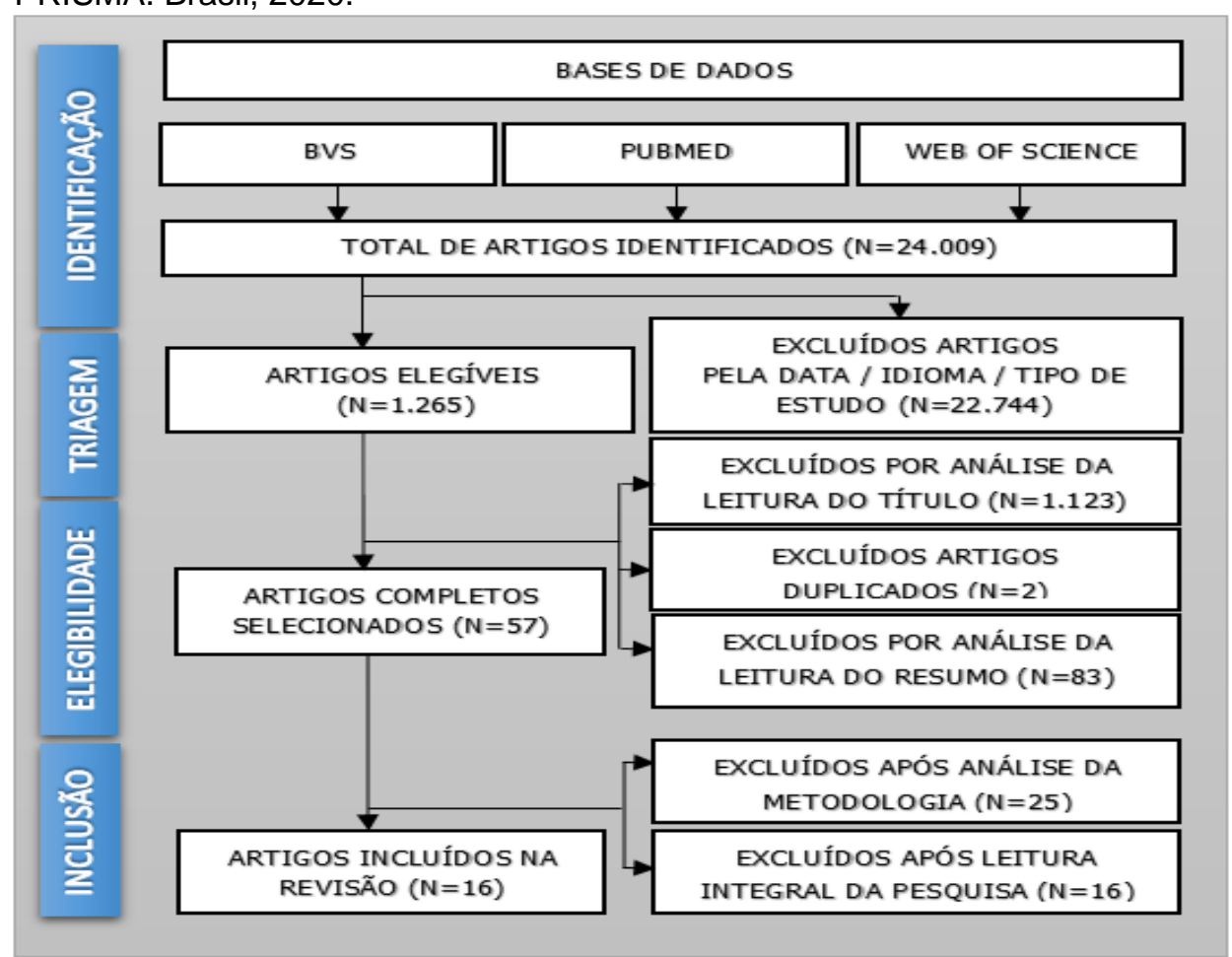

Fonte: Costa TRM, et al., 2020.

\section{RESULTADOS}

Os estudos científicos que participaram desta revisão sistemática encontraram-se entre os anos de 2010 a 2019. Através da seleção do banco de dados, 24.009 artigos foram identificados sobre o tema de interesse. Após a remoção de 22.774 artigos pelo ano, idioma que não fossem português, inglês ou espanhol e estudos diferentes de ensaios clínicos, 1.265 artigos foram obtidos para a realização da análise. Consecutivamente, com uma verificação dos títulos mais abrangentes, dos artigos duplicados e dos resumos dos artigos acarretou a eliminação de 1.208 artigos, resultando em 57 artigos no primeiro estágio do estudo e elegíveis para a segunda etapa da revisão sistemática. Dessa forma, dos 57 estudos analisados na íntegra, 40 foram excluídos pelos seguintes motivos: 25 artigos não atingiram uma boa qualidade segundo a Escala de Jadad (Avaliação de Ensaio-Clínico), com score menor que quatro, e 16 encaixaram-se nos critérios de exclusão devido aos dados não contemplarem o objetivo de pesquisa. Por fim, obteve-se 16 estudos incluídos na revisão. $O$ índice de concordância entre os pesquisadores foi considerado como aceitável $(0,8251)$.

A média da população dos 16 artigos, desta revisão, foi de 3.730,87 pacientes, em 13 pesquisas o método de Intervenção Coronária Percutânea (IPC) foi realizado, ao passo que em oito (8) utilizou-se o método da Cirurgia de Revascularização Miocárdica (CRM), sendo que destes dois (2) aplicaram a técnica na presença ou ausência de circulação extracorpórea. No que se refere às complicações verificadas nos desfechos clínicos dos ensaios, constatou-se que o IAM foi complicação em 11 estudos, acidente vascular cerebral (AVC) em nove (09), eventos hemorrágicos em oito (08), seis (06) estudos apontaram a morte pós-operatória e cinco (05) a morte cardíaca como eventuais complicações dos métodos de revascularização cardíaca.

Além dessas, outras complicações encontradas nos artigos com menor frequência foram: trombose de stent (três), fibrilação atrial (dois), revascularização de lesão com indicação clínica (dois), insuficiência cardíaca (IC) (dois), nefropatia por uso de contraste (dois), arritmia (um), choque cardiogênico (um), falha da lesão alvo (um), angina instável (um), tamponamento cardíaco (um), taquicardia ventricular por trombo intracoronário (um) e infecção (um). Dentre as publicações incluídas, abordaram-se os principais achados dos estudos, contendo algumas características dos ensaios clínicos obtidos nesta presente pesquisa: o título do artigo, os autores, o tamanho amostral, os procedimentos cirúrgicos realizados e as complicações identificadas, esses estão expressos abaixo (Quadro 1). 
Quadro 1 - Artigos selecionados para leitura e análise (2010 - 2019).

\begin{tabular}{|c|c|c|c|c|}
\hline Artigo & Autores & Amostra & Procedimentos cirúrgicos & Complicações \\
\hline $\begin{array}{l}\text { 1- Characteristics and prognosis of coronary } \\
\text { revascularization procedure in patients age } 80 \text { and older } \\
\text { presenting with acute myocardial infarction. }\end{array}$ & $\begin{array}{l}\text { Mansencal N, } \\
\text { et al., } \\
2010 .\end{array}$ & $\begin{array}{l}1.687 \\
\text { pacientes }\end{array}$ & ICP & $\begin{array}{l}\text { Choque cardiogênico; Morte intra- } \\
\text { hospitalar. }\end{array}$ \\
\hline $\begin{array}{l}\text { 2- No Major Differences in 30-Day Outcomes in High-Risk } \\
\text { Patients Randomized to Off-Pump Versus On-Pump } \\
\text { Coronary Bypass Surgery The Best Bypass Surgery Trial. }\end{array}$ & $\begin{array}{l}\text { Møller } \mathrm{CH} \text {, et } \\
\text { al., } 2010 .\end{array}$ & 341 pacientes & $\begin{array}{l}\text { CRM (com ou sem } \\
\text { circulação extracorpórea) }\end{array}$ & Fibrilação atrial. \\
\hline $\begin{array}{l}\text { 3- Impact of Bivalirudin Therapy in High-Risk Patients With } \\
\text { Acute Myocardial Infarction }\end{array}$ & $\begin{array}{l}\text { Parodi G, et } \\
\text { al., } 2010 .\end{array}$ & $\begin{array}{l}2.530 \\
\text { pacientes }\end{array}$ & ICP & $\begin{array}{l}\text { Mortalidade pós-operatória; Infarto } \\
\text { agudo do miocárdio recorrente. }\end{array}$ \\
\hline $\begin{array}{c}\text { 4- The Impact of Patient and Lesion Complexity on Clinical } \\
\text { and Angiographic Outcomes After Revascularization With } \\
\text { Zotarolimus- and Everolimus-Eluting Stents. }\end{array}$ & $\begin{array}{l}\text { Stefanini GG, } \\
\text { et al., } 2011 .\end{array}$ & $\begin{array}{c}2.292 \\
\text { pacientes }\end{array}$ & ICP & $\begin{array}{c}\text { Falha da lesão alvo; Morte cardíaca; } \\
\text { Infarto agudo do miocárdio; } \\
\text { Revascularização de lesão clinicamente } \\
\text { indicada; Trombose definitiva ou } \\
\text { provável do stent. }\end{array}$ \\
\hline $\begin{array}{l}\text { 5- Radial Versus Femoral Randomized Investigation in ST- } \\
\text { Segment Elevation Acute Coronary Syndrome. }\end{array}$ & $\begin{array}{l}\text { Romagnoli E, } \\
\text { et al., } 2012 .\end{array}$ & $\begin{array}{c}1.001 \\
\text { pacientes }\end{array}$ & ICP & $\begin{array}{l}\text { Mortalidade cardíaca; Eventos } \\
\text { hemorrágicos. }\end{array}$ \\
\hline $\begin{array}{l}\text { 6- Early Aggressive Versus Initially Conservative Treatment } \\
\text { in Elderly Patients With Non-ST-Segment Elevation Acute } \\
\text { Coronary Syndrome A Randomized Controlled Trial. }\end{array}$ & $\begin{array}{l}\text { Savonitto S, et } \\
\text { al., } 2012 \text {. }\end{array}$ & 313 pacientes & CRM e ICP & $\begin{array}{l}\text { Morte; Infarto agudo do miocárdio; } \\
\text { Repetição do internamento por eventos } \\
\text { hemorrágicos. }\end{array}$ \\
\hline $\begin{array}{l}\text { 7- Factors Contributing to the Lower Mortality With } \\
\text { Ticagrelor Compared With Clopidogrel in Patients } \\
\text { Undergoing Coronary Artery Bypass Surgery. }\end{array}$ & $\begin{array}{l}\text { Varenhorst C, } \\
\text { et al., } 2012 .\end{array}$ & $\begin{array}{c}18.624 \\
\text { pacientes }\end{array}$ & CRM & $\begin{array}{l}\text { Infarto do miocárdio, insuficiência } \\
\text { cardíaca, morte súbita, arritmia e } \\
\text { acidente vascular cerebral, eventos } \\
\text { hemorrágicos, infecção }\end{array}$ \\
\hline $\begin{array}{l}\text { 8- Impact of Multiple Complex Plaques on Short- And Long- } \\
\text { Term Clinical Outcomes in Patients Presenting With ST- } \\
\text { segment Elevation Myocardial Infarction. }\end{array}$ & $\begin{array}{c}\text { Keeley EC, et } \\
\text { al., } 2014 .\end{array}$ & $\begin{array}{c}3.467 \\
\text { pacientes }\end{array}$ & ICP & $\begin{array}{c}\text { Reinfarto, revascularização de lesão } \\
\text { alvo acionada clinicamente, acidente } \\
\text { vascular cerebral, morte cardíaca e } \\
\text { sangramento }\end{array}$ \\
\hline $\begin{array}{l}\text { 9- Complete revascularisation versus treatment of the } \\
\text { culprit lesion only in patients with ST-segment elevation } \\
\text { myocardial infarction and multivessel disease: an open- } \\
\text { label, randomised controlled trial. }\end{array}$ & $\begin{array}{l}\text { Engstrøm T, et } \\
\text { al., } 2015 .\end{array}$ & 627 pacientes & ICP & $\begin{array}{l}\text { Infarto do miocárdio, sangramento que } \\
\text { requer transfusão, nefropatia induzida } \\
\text { por contraste, acidente vascular } \\
\text { cerebral }\end{array}$ \\
\hline
\end{tabular}

REAS/EJCH | Vol.12(11) | e4834 | DOI: https://doi.org/10.25248/reas.e4834.2020 Página 6 de 11 


\begin{tabular}{|c|c|c|c|c|}
\hline Artigo & Autores & Amostra & Procedimentos cirúrgicos & Complicações \\
\hline $\begin{array}{l}\text { 10- Efficacy and Safety of Cangrelor in Preventing } \\
\text { Periprocedural Complications in Patients With Stable } \\
\text { Angina and Acute Coronary Syndromes Undergoing } \\
\text { Percutaneous Coronary Intervention The CHAMPION } \\
\text { PHOENIX Trial. }\end{array}$ & $\begin{array}{l}\text { Abtan J, et al., } \\
2016 .\end{array}$ & $\begin{array}{c}10.942 \\
\text { pacientes }\end{array}$ & ICP & $\begin{array}{l}\text { Morte, infarto agudo do miocárdio, } \\
\text { revascularização induzida por eventos } \\
\text { isquêmicos ou trombose de stent }\end{array}$ \\
\hline $\begin{array}{l}\text { 11- Coronary Artery Bypass Grafting Versus Drug-Eluting } \\
\text { Stents Implantation for Previous Myocardial Infarction. }\end{array}$ & $\begin{array}{l}\text { Chang } M \text {, et } \\
\text { al., } 2016 .\end{array}$ & $\begin{array}{l}3.280 \\
\text { pacientes }\end{array}$ & CRM e ICP & $\begin{array}{l}\text { Morte pós-operatória por qualquer } \\
\text { causa, infarto do miocárdio, acidente } \\
\text { vascular cerebral }\end{array}$ \\
\hline $\begin{array}{l}\text { 12-A Randomized Trial of Complete Versus Culprit-Only } \\
\text { Revascularization During Primary Percutaneous Coronary } \\
\text { Intervention in Diabetic Patients With Acute ST Elevation } \\
\text { Myocardial Infarction and Multi Vessel Disease. }\end{array}$ & $\begin{array}{l}\text { Hamza M, et } \\
\text { al., } 2016 .\end{array}$ & 100 pacientes & ICP & $\begin{array}{l}\text { Acidente vascular cerebral, nefropatia } \\
\text { induzida por contraste, sangramento } \\
\text { maior ou menor }\end{array}$ \\
\hline $\begin{array}{l}\text { 13- Outcomes of Patients Receiving Downstream } \\
\text { Revascularization After Initial Medical Management for } \\
\text { Non-ST-Segment Elevation Acute Coronary Syndromes. }\end{array}$ & $\begin{array}{l}\text { Hinohara TT, } \\
\text { et al., } 2018 .\end{array}$ & $\begin{array}{c}9.326 \\
\text { pacientes. }\end{array}$ & CRM e ICP & $\begin{array}{c}\text { Eventos hemorrágicos, morte } \\
\text { cardiovascular, infarto miocárdico não } \\
\text { fatal, acidente vascular cerebral não } \\
\text { fatal }\end{array}$ \\
\hline $\begin{array}{l}\text { 14- Ten-Year Follow-Up of Off-Pump and On-Pump } \\
\text { Multivessel Coronary Artery Bypass Grafting: MASS III. }\end{array}$ & $\begin{array}{l}\text { Hueb W, et al., } \\
2019 .\end{array}$ & 308 pacientes & $\begin{array}{l}\text { CRM (com ou sem } \\
\text { circulação extracorpórea) }\end{array}$ & $\begin{array}{c}\text { Infarto do miocárdio, acidente vascular } \\
\text { cerebral, revascularização adicional e } \\
\text { complicações pós-operatórias, fibrilação } \\
\text { atrial }\end{array}$ \\
\hline $\begin{array}{l}\text { 15- Complete Revascularization with Multivessel PCI for } \\
\text { Myocardial Infarction. }\end{array}$ & $\begin{array}{l}\text { Mehta SR, et } \\
\text { al., } 2019 .\end{array}$ & $\begin{array}{c}4.041 \\
\text { pacientes }\end{array}$ & CRM e ICP & $\begin{array}{c}\text { Morte por causas cardiovasculares, } \\
\text { novo infarto do miocárdio, } \\
\text { revascularização induzida por isquemia, } \\
\text { angina instável, insuficiência cardíaca } \\
\text { (classe IV, NY heart association), } \\
\text { hemorragia grave, acidente vascular } \\
\text { cerebral, trombose de stent }\end{array}$ \\
\hline $\begin{array}{c}\text { 16- Randomized Trial Evaluating Percutaneous } \\
\text { Coronary Intervention for the Treatment of Chronic Total } \\
\text { Occlusion The DECISION-CTO Trial. }\end{array}$ & $\begin{array}{l}\text { LEE SW, et } \\
\text { al., } 2019 .\end{array}$ & 815 pacientes & $\mathrm{ICP}$ & $\begin{array}{l}\text { Acidente vascular cerebral, } \\
\text { tamponamento cardíaco, taquiarritmia } \\
\text { ventricular induzida por trombo } \\
\text { intracoronário }\end{array}$ \\
\hline
\end{tabular}

Fonte: Costa TRM, et al., 2020. 


\section{DISCUSSÃO}

As complicações dos métodos de revascularização cardíaca em pacientes que sofreram IAM foram identificadas de maneira ampla e diversificada na análise dos artigos. Os artigos encontrados abordaram técnicas como os stents coronarianos, a revascularização com ou sem a utilização da circulação extracorpórea e a implementação medicamentosa, como forma de minimizar os riscos, além de abordar a maneira adequada de avaliação de risco dos pacientes.

Foi perceptível que as complicações podem apresentar-se de diversas formas, incluindo: a fibrilação atrial, morte súbita, disritmias ventriculares, insuficiência cardíaca, processos infecciosos e infarto do miocárdio (ABTAN J, et al., 2016; HUEB W, et al., 2019). As mais frequentes foram o Acidente Vascular Cerebral (AVC) e eventos hemorrágicos seguidos por morte pós-operatória, sendo classificadas de acordo com Varenhorst C, et al. (2012), em vascular e não vascular com base na causa do óbito.

Os fatores intrínsecos ligados aos riscos de complicações são avaliados através do Sistema Europeu de Avaliação de Risco Operacional Cardíaco (Euro SCORE), o qual é responsável pela classificação em grupos, sendo em três: baixo, médio e alto risco, sua importância é devido a padronização da classificação.

Essa estratificação é baseada em idade, fração de ejeção, disfunção renal, doença respiratória, doença cerebrovascular, história prévia de cirurgia cardíaca, estado crítico pré-operatório, angina instável, hipertensão pulmonar, cirurgia de emergência, infarto do miocárdio recente, ruptura do septo ventricular, endocardite ativa, doença vascular periférica, cirurgia aórtica torácica.

O modelo foi utilizado em um dos estudos apresentados de forma avaliativa aos pacientes submetidos a cirurgia de revascularização com ou sem a utilização da bomba de circulação extracorpórea (MØLLER CH, et al., 2010).

A cirurgia de revascularização com a bomba de Circulação Extracorpórea (CEC) faz com que o sangue seja desviado do coração, como também dos pulmões no período operatório. Apesar de ser considerada uma técnica reconhecida e acarretar maior conforto aos profissionais da saúde, há um maior risco de complicações como infecções por conta do contato do sangue com materiais estranhos (DIENSTMANN C e CAREGNATO RCA, 2013; MOTA AL, et al., 2008).

No entanto, no estudo realizado por Møller $\mathrm{CH}$, et al. (2010), com 341 pacientes de alto risco, foi observado que a cirurgia de revascularização, com ou sem a utilização da bomba, em ambos os enxertos teve alta eficácia, com baixas complicações de curto prazo, como foi a fibrilação atrial, ocorrendo em quase metade dos pacientes nos dois grupos.

A evolução das técnicas de revascularização tem como propósito diminuir os riscos cirúrgicos e complicações. Atrelado a isso, as medidas no manejo pré e pós-cirúrgicos são imprescindíveis para evitar maiores danos.

Esse preparo é através da utilização de fármacos, novos dispositivos e métodos cirúrgicos, alguns já citados, como a utilização de enxertos com ou sem CEC. A melhor abordagem, seja para reverter ou prevenir a situação do paciente, a qual dependerá da complexidade, determinará um prognóstico e o tipo de complicação (BRAILE DM, 2010; STEFANINI GG, et al., 2011).

O Synergy Between Percutaneous Coronary Intervention with Taxus and Cardiac Surgery (SYNTAX) é um score diferente do EuroSCORE, pois avalia as características da Doença Arterial Coronariana (DAC) em cada paciente, com o objetivo de identificar a extensão e a complexidade de lesões arteriosclerótica (STEFANINI GG, et al., 2011; BRAILE DM e GOMES WJ, 2010).

O intuito maior para aplicação dos scores é definir a melhor terapia de forma individualizada, ponderando os riscos e complicações em cada paciente, logo que os eventos adversos cardiovasculares são mais comuns em pacientes complexos em comparação aos simples (STEFANINI GG, et al., 2011). Entretanto, há exceções a essa estatística, ao comparar a utilização de dois stents de nova geração como os de Zetarolimus (ZES) e os de Eluição de Everolimus (EES), cujos ambos se mostraram eficazes e seguros, independente das complicações clínicas dos pacientes ao longo de 1 ano. Eles estão associados as baixas taxas de 
complicações relacionadas ao segmento ST em populações complexas e simples de pacientes (STEFANINI GG, et al., 2011).

Em torno dos tratamentos farmacológicos, obtiveram boas respostas os antiagregantes plaquetários e os anticoagulantes, med iante a revascularização, para o aumento da sobrevida e redução das complicações. Em relação a primeira classe, respectivamente, foi feito um estudo comparativo entre o Ticagrelor e o Clopidogrel. Foi observado que as complicações de causa direta como sangramento e eventos infecciosos, após a revascularização, foram mais presentes em pacientes submetidos ao Ticagrelor comparado ao Clopidogrel.

Por fim, o grupo dos anticoagulantes como a Bivalirudina, o qual demonstrou-se uma maior eficácia na redução da mortalidade, frente a Heparina não-fracionada (HNF) mais Inibidores de glicoproteína opcionais (GPI) em pacientes de alto risco submetidos a intervenção percutânea primária no tratamento agudo do IAM (VARENHORST C, et al., 2012; PARODI G, et al., 2010).

Estudos também demonstraram a alta sensibilidade da angiografia coronariana em comparação com outras investigações cardíacas, e o benefício de revascularização do miocárdio (CRM), antes da cirurgia periférica revascularização, tendo em vista que a angiografia identifica qualquer estenose coronariana seja severa ou não (ILLUMINATI G, et al., 2010).

A melhora da técnica do cateterismo da artéria coronária e a disponibilidade contemporânea de coronárias stent sobre a CRM poderia, teoricamente, apoiar o desempenho da angiografia coronariana e, possivelmente, como parte do protocolo pré-operatório padrão para pacientes programados para a revascularização arterial periférica.

As evidências encontradas apoiam a hipótese de que realizando angiografia coronária pré-operatória seguida, se necessário, pela ICP antes da Endarterectomia de Carótida (CEA), reduziu significativamente a incidência de isquemia cardíaca pós-operatória, eventos sem qualquer morbidade adicional (ILLUMINATI G, et al., 2010). O manejo correto das complicações é imprescindível para a recuperação do paciente. A fibrilação atrial aguda no pós-operatório tem como objetivo reversão a um ritmo sinusal em 24 horas com uso de drogas, por exemplo, a amiodarona.

Já a hipotensão no pós-operatório que leva a síndrome vasoplegica é revertida com reposição volêmica adequada associada a noradrenalina em diluição padrão em Unidades de Terapia Intensiva (UTI)

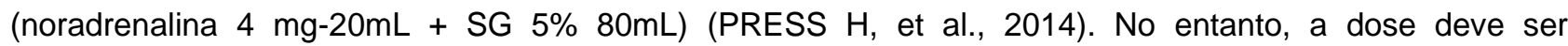
individualizada para cada paciente, já que em alguns podem requerer a associação com outras drogas.

No caso do sangramento, o qual é definido com perda maior que $3 \mathrm{ml} / \mathrm{kg} / \mathrm{hora}$ nas primeiras 3 horas ou maior que $1,5 \mathrm{ml} / \mathrm{Kg} /$ hora após a $3^{a}$ hora de pós-operatório, deve-se sempre na presença de sangramento acima do normal, contactar a equipe cirúrgica, pois poderá ser necessário a reexploração, junto a coleta de coagulograma completo (NASCIMENTO BR, et al., 2018; PRESS H, et al., 2014).

Apesar da presença de limitações sobre o benefício da revascularização, frente aos possíveis danos ao miocárdio, a literatura compara os efeitos da CRM e ICP com stent farmacológico, demonstrando a eficácia desse procedimento, como abordagem ideal em pacientes com infarto do miocárdio prévio e DAC esquerda ou principal e multifatorial. Assim, reduzindo a mortalidade drasticamente nesses casos (NASCIMENTO BR, et al., 2018; CHANG M, et al., 2016).

\section{CONSIDERAÇÕES FINAIS}

Por fim, a partir do estudo, foi possível identificar e compreender as complicações apresentadas por pacientes Pós-Infarto Agudo do Miocárdio (IAM), submetidos a diferentes métodos de revascularização cardíaca como opção terapêutica. As complicações apresentam-se como fibrilação atrial, morte súbita, disritmias ventriculares, insuficiência cardíaca, processos infecciosos e infarto do miocárdio, além do Acidente Vascular Cerebral (AVC) e eventos hemorrágicos seguidos por morte pós-operatória. Portanto, é imprescindível que a medicina utilize novas técnicas com o propósito de diminuir os riscos cirúrgicos, além de obter auxílio com scores para avaliar a classificação de risco, assim alcançando um tratamento adequado. 


\section{REFERÊNCIAS}

1. ABTAN J, et al. Efficacy and Safety of Cangrelor in Preventing Periprocedural Complications in Patients With Stable Angina and Acute Coronary Syndromes Undergoing Percutaneous Coronary Intervention: The CHAMPION PHOENIX Trial. JACC: Cardiovascular Interventions, 2016; 9(18): 1905-1913.

2. ANDRADE JP, de et al. IV Diretriz da Sociedade Brasileira de Cardiologia sobre tratamento do infarto agudo do miocárdio com supradesnível do segmento ST. Arq Bras Cardiol, 2009; 93(6): 179-264.

3. AVEZUM Á, et al. III Diretriz sobre tratamento do infarto agudo do miocárdio. Arquivos Brasileiros de Cardiologia, 2004; 83: 1-86.

4. BRAILE DM, GOMES WJ. Evolução da cirurgia cardiovascular: a saga brasileira. Uma história de trabalho, pioneirismo e sucesso. Arq Bras Cardiol, 2010; 94(2): 151-2.

5. CANTOR AB. Sample-size calculations for Cohen's Kappa. Psychol Methods. 1996;1(2):150-3.

6. CHANG M, et al. Coronary Artery Bypass Grafting Versus Drug-Eluting Stents Implantation for Previous Myocardial Infarction. The American journal of cardiology, 2016; 118(1): 17-22.

7. BRICK AV, et al. Diretrizes da cirúrgia de revascularização miocárdica valvopatias e doenças da aorta. Arquivos Brasileiros de Cardiologia, 2004; 82; 1-20.

8. DE CARVALHO ACA, et al. Desenvolvimento de placas de ateroma em pacientes diabéticos e hipertensos. Revista de Ciências Médicas e Biológicas, 2010; 9(1): 73-77.

9. DE SOUZA AR, et al. Fatores associados a um impacto na qualidade de vida pós revascularização miocárdica. Revista Rene UFC, 2018: 1-10.

10. DI CASTRO CURADO FAM et al. Intervenção coronária percutânea primária ou de resgate em tabagistas. Revista Brasileira de Cardiologia Invasiva, 2016; 24(4): 19-24.

11. DIENSTMANN C, CAREGNATO RCA. Circulação Extracorpórea em cirurgia cardíaca: um campo de trabalho para o enfermeiro. Rev SOBECC. 2013; 18(1): 35-43.

12. ENGSTRØM T, et al. Complete revascularisation versus treatment of the culprit lesion only in patients with STsegment elevation myocardial infarction and multivessel disease (DANAMI-3-PRIMULTI): an open-label, randomised controlled trial. The Lancet, 2015; 386(9994): 665-671.

13. FEITOSA ACR, et al. II Diretriz de avaliação perioperatória da Sociedade Brasileira de Cardiologia. Arquivos Brasileiros de Cardiologia, 2011; 96(3): 1-68.

14. FERES F, et al. Diretriz da sociedade brasileira de cardiologia e da sociedade brasileira de hemodinâmica e cardiologia intervencionista sobre intervenção coronária percutânea. Arquivos Brasileiros de Cardiologia, 2017; 109(1): 1-81.

15. GODOY MF, et al. Mortalidade por doenças cardiovasculares e níveis socioeconômicos na população de São José do Rio Preto, estado de São Paulo, Brasil. Arquivos Brasileiros de Cardiologia, 2007; 88(2): 200-206.

16. HAMZA M, et al. A Randomized Trial of Complete Versus Culprit-Only Revascularization During Primary Percutaneous Coronary Intervention in Diabetic Patients With Acute ST Elevation Myocardial Infarction and Multi Vessel Disease. Journal of Interventional Cardiology, 2016; 29(3): 241-247.

17. HINOHARA TT, et al. Outcomes of Patients Receiving Downstream Revascularization After Initial Medical Management for Non-ST-Segment Elevation Acute Coronary Syndromes (From the TRILOGY ACS Trial). The American journal of cardiology, 2018;122(8):1322-1329.

18. HUEB W, et al. Ten-Year Follow-Up of Off-Pump and On-Pump Multivessel Coronary Artery Bypass Grafting: MASS III. Angiology, 2019; 70(4): 337-344.

19. ILLUMINATI G, et al. Systematic preoperative coronary angiography and stenting improves postoperative results of carotid endarterectomy in patients with asymptomatic coronary artery disease: a randomised controlled trial. European journal of vascular and endovascular surgery: the official journal of the European Society for Vascular Surgery, 2010; 39(2): 139-145.

20. JADAD AR, et al. Assessing the quality of reports of randomized clinical trials: is blinding necessary? Control Clin Trials. 1996; 17: 1-12.

21. KEELEY EC, et al. Impact of multiple complex plaques on short- and long-term clinical outcomes in patients presenting with ST-segment elevation myocardial infarction (from the Harmonizing Outcomes With Revascularization and Stents in Acute Myocardial Infarction [HORIZONS-AMI] Trial). The American journal of cardiology, 2014; 113(10): $1621-1627$.

22. LEE SW, et al. Randomized Trial Evaluating Percutaneous Coronary Intervention for the Treatment of Chronic Total Occlusion. Circulation, 2019; 139(14): 1674-1683.

23. MAGALHÃES FJ, et al. Risk factors for cardiovascular diseases among nursing professionals: strategies for health promotion. Revista Brasileira de Enfermagem, 2014; 67(3) 394-400.

24. MANSENCAL N, et al. Characteristics and prognosis of coronary revascularization procedure in patients age 80 and older presenting with acute myocardial infarction. Medical science monitor: international medical journal of experimental and clinical research, 2009; 16(1): 15-20.

25. MEHTA SR, et al. Complete Revascularization with Multivessel PCI for Myocardial Infarction. The New England journal of medicine, 2019; 381(15): 1411-1421.

26. MIRANDA GMD, et al. O envelhecimento populacional brasileiro: desafios e consequências sociais atuais e futuras. Revista Brasileira de Geriatria e Gerontologia, 2016; 19(3): 507-519.

27. MOTA AL, et al. Circulação extracorpórea em adultos no século XXI. Ciência, arte ou empirismo? Brazilian J Cardiovasc Surg. 2008; 23(1):78-92.

28. MØLLER CH, et al. No major differences in 30-day outcomes in high-risk patients randomized to off-pump versus onpump coronary bypass surgery: the best bypass surgery trial. Circulation, 2010; 121(4): 498-504. 
29. NASCIMENTO BR, et al. Cardiovascular disease epidemiology in Portuguese-Speaking countries: data from the Global Burden of Disease, 1990 to 2016. Arquivos brasileiros de cardiologia, 2018; 110(6): 500-511.

30. PARODI G, et al. Impact of bivalirudin therapy in high-risk patients with acute myocardial infarction: 1-year results from the HORIZONS-AMI (Harmonizing Outcomes with RevasculariZatiON and Stents in Acute Myocardial Infarction) trial. JACC. Cardiovascular interventions, 2010; 3(8): 796-802.

31. PIEGAS LS; HADDAD N. Intervenção coronariana percutânea no Brasil: resultados do Sistema Único de Saúde. Arquivos Brasileiros de Cardiologia, 2011; 96(4): 317-324.

32. PIEGAS LS, et al. V Diretriz da Sociedade Brasileira de Cardiologia sobre tratamento do infarto agudo do miocárdio com supradesnível do segmento ST. Arquivos brasileiros de cardiologia, 2015; 105(2): 1-121.

33. PRESS H. Protocolo de condutas no pós operatório do Serviço de Cirurgia Cardíaca do HUCFF - ICES, UFRJ. Objetivos hemodinâmicos no pós operatório Reposição volêmica no pós operatório Tratamento da fibrilação atrial aguda no pós operatório Hipotensão no Pós Operatório / Síndrome Vasoplégica Síndrome de Baixo Débito Cardíaco no Pós Operatório, 2014.

34. ROMAGNOLI E, et al. Radial Versus Femoral Randomized Investigation in ST-Segment Elevation Acute Coronary Syndrome: The RIFLE-STEACS (Radial Versus Femoral Randomized Investigation in ST-Elevation Acute Coronary Syndrome) Study. Journal of the American College of Cardiology, 2012; 60(24): 2481-2489.

35. SANTOS J, et al. Mortalidade por infarto agudo do miocárdio no Brasil e suas regiões geográficas: análise do efeito da idade-período-coorte. Ciência \& Saúde Coletiva, 2018; 23: 1621-1634.

36. SAVONITTO S, et al. Early Aggressive Versus Initially Conservative Treatment in Elderly Patients With Non-STSegment Elevation Acute Coronary Syndrome. JACC: Cardiovascular Interventions, 2012; 5(9): 906-916.

37. SIERVULI MTF, et al. Infarto do miocárdio: alterações morfológicas e breve abordagem da influência do exercício físico. Rev Bras Cardiol, 2014; 27(5): 349-55.

38. STEFANINI GG, et al. The impact of patient and lesion complexity on clinical and angiographic outcomes after revascularization with zotarolimus- and everolimus-eluting stents: a substudy of the RESOLUTE All Comers Trial (a randomized comparison of a zotarolimus-eluting stent with an everolimus-eluting stent for percutaneous coronary intervention). Journal of the American College of Cardiology, 2011; 57(22): 2221-2232.

39. VARENHORST C, et al. Factors contributing to the lower mortality with ticagrelor compared with clopidogrel in patients undergoing coronary artery bypass surgery. Journal of the American College of Cardiology, 2012; 60(17): 1623-1630. 